\title{
Improving access and quality of care in a TB control programme
}

\author{
Vera Scott, Virginia Azevedo, Judy Caldwell
}

Objectives. To use a quality improvement approach to improve access to and quality of tuberculosis (TB) diagnosis and care in Cape Town.

Methods. Five HIV/AIDS/sexually transmitted infections/TB (HAST) evaluations were conducted from 2008 to 2010, with interviews with 99 facility managers and a folder review of over 850 client records per evaluation cycle. The data were used in a local quality improvement process: sub-district workshops identified key weaknesses and facility managers drew up action plans. Lessons learnt and successful strategies were shared at quarterly districtwide HIV/TB meetings.

Results. Geographical access was good, but there were delays in treatment commencement times. Access for high-risk clients improved significantly with intensified TB case finding made routine in both the HIV counselling and testing and antiretroviral treatment (ART) services ( $p<0.01$ for both). Access for children in contact with an infectious case has improved but is still low ( $42 \%$ investigated and treated). Quality of care was mostly high at baseline (adherence to treatment protocols 95\%). Measurement of body mass index improved from $20 \%$ to $62 \%$. The assessment of contraception improved from $27 \%$ to $58 \%$. Care for co-infected clients showed improved use of customised HIV stationery and increased assessment for ART eligibility.

Conclusions. The HAST audit contributed to the improved TB cure rates by supplementing routine information and involving sub-district managers, facility managers and facility staff in a quality improvement process that identified local opportunities for programme strengthening.

S Afr Med J 2012;102(11):837-840. DOI:10.7196/SAMJ.5469
South Africa has the second-highest tuberculosis (TB) incidence in the world. ${ }^{1}$ Driven by the emerging HIV epidemic, the incidence rose from 332 to $970 / 100000$ population between 1996 and 2009, and is now complicated by the emergence of drug-resistant TB. Health services in South Africa struggled to respond effectively to the increased case burden. Despite early attempts to provide additional support, ${ }^{2}$ the cure rates were initially modestly improved from $53.9 \%$ to $57.7 \%$ from 1996 to $2005,{ }^{3}$ which was well below the target of $85 \%$. A regional declaration in 2005 recognised TB as an emergency, and national crisis plans were developed to support poorly performing districts with high case loads. Implementation weaknesses were recognised within the TB programme ${ }^{1}$ and the public primary care service, ${ }^{4}$ resulting in calls for functional district health systems and innovation. ${ }^{5}$ The Tuberculosis Strategic Plan for South Africa, 2007 - 2011, ${ }^{3}$ sets out measurable objectives and targets to be achieved by 2011, including a case detection rate of $70 \%$, a cure rate of $85 \%$, and targets that are specifically for HIV/TB integration.

In Cape Town, the cure rate for new smear-positive clients increased from $67 \%$ to $80 \%$ (2004 - 2009); one sub-district improved from $52 \%$ to $78 \%$. This remarkable success has been supported by a number of interventions. Firstly, there has been additional funding for the TB programme to employ more nursing staff and to create two new cadres at most of the high-burden clinics - TB clerks responsible for programme administration and $\mathrm{TB}$ assistants who make home visits to recall sputum-positive $\mathrm{TB}$ suspects and trace defaulters. Secondly, monitoring and

School of Public Health, University of the Western Cape, Bellville, Western Cape Vera Scott, MB ChB, DCH, MPH

Department of Health, City of Cape Town

Virginia Azevedo, MB ChB, BSc (Med) (Hons) (Community Health)

Judy Caldwell, BA (Cur) (Hons)

Corresponding author: Vera Scott (verascott@mweb.co.za) evaluation has been improved with quarterly HIV/TB/sexually transmitted infection (STI) reviews attended by sub-district and facility staff; routine data were used to identify and support poorly performing facilities. Thirdly, driving the integration of HIV and TB services at the point of patient care has brought about increased training and mentoring on local protocols, and increased resources through linkages with the antiretroviral treatment (ART) programme. Fourthly, performance management has been implemented for sub-district managers, prioritising the TB programme. This has encouraged sub-district managers, who manage implementation of the programmes at sub-district and facility level, to actively manage the TB programme.

We focus on the fifth intervention, the objective of which was to use a quality improvement approach to improve access to and quality of TB diagnosis and care. Integrated HIV/TB/STI audit tools were developed between 2003 and $2006^{6}$ and routinely implemented in all 146 public primary care facilities in Cape Town in 2007. The clinical audit emphasised reflective practice and using health information as a management tool at sub-district and facility level to improve service delivery. Five audits were done between 2008 and 2010; they identified further necessary improvements in access and quality and strategies were put in place.

The Cape Town Metro Health District has a population of approximately 3.8 million, and is divided into 8 health sub-districts. During the study, each sub-district had 2 HIV/AIDS/STI/TB (HAST) co-ordinators who provided programme support to the primary care facilities. Primary services were offered by the local and provincial authorities, who collaborate to ensure efficient service delivery. All 146 facilities offered TB diagnosis, HIV counselling and testing (HCT) and care for HIV-positive clients who were not yet eligible for ART (pre-ART); 99 facilities offered TB treatment and 51 offered ART.

Facilities that treated TB had a designated TB treatment room, and those with a high annual TB case load ( $>200$ clients registered per year) had staff assigned to manage the TB programme. The TB staff were also responsible for identifying child contacts $(<5$ years of age $)$ of infectious cases, investigating them and commencing prophylaxis or treatment. 
In Cape Town, most HIV-positive clients were identified through HCT and directed to one of two management packages: pre-ART clients (i.e. receiving ongoing monitoring and care before being eligible for ART) or ART clients. Clients were screened at each of these service points for TB symptoms.

\section{Methods}

The study design is a participatory programme evaluation aimed at improving service delivery. Data were collected in 5 audit cycles between 2008 and 2010 using the Integrated HAST Evaluation tools (available from authors), consisting of an extract of routine data, a facility manager questionnaire, observations and a set of TB, HCT, HIV, ART and prevention of mother-to-child transmission (PMTCT) folder reviews. All public primary care facilities in Cape Town were eligible. Each audit cycle was preceded by a 1-day training workshop based on a field manual and detailing the rationale and methods. These were led by district programme managers and attended by the 16 sub-district HAST co-ordinators, sub-district supervisors, facility managers and family physicians responsible for clinical governance. The HAST co-ordinators of the 8 sub-districts were responsible for drawing together their own audit teams (approximately 4 teams per sub-district, with 4 - 6 team members each, including subdistrict managers, facility managers and facility staff working in the programmes), providing additional on-site training as required, and organising the logistics of the facility visits in their sub-districts, which were to be completed within 1 month of the start of the audit cycle.

It took approximately 3 hours in each facility to interview the facility manager, observe the equipment in consulting and counselling rooms, and conduct folder reviews. Ten TB folders in each facility were randomly sampled; we worked backwards from 4 months before (allowing time for patients to receive aspects of care that were being assessed). A similar procedure was adopted to sample HCT folders and ART folders. Routine data were drawn from the electronic TB register.

Facility data were entered in Excel spreadsheets and imported into STATA version 9 for calculations of district proportions, confidence intervals (CIs) for sampled data, and tests of statistical significance. Because proportional sampling had not been done for the folder reviews, a weighting factor was introduced, namely the proportion of the total annual TB case load in each facility.
Workshops were held in the 8 sub-districts to analyse the data. Facility managers were responsible for drawing up action plans to address key problems and were accountable to their primary care supervisors for implementing these plans. Lessons learnt and successful strategies were shared at quarterly district-wide HIV/TB meetings.

The study was approved by the Western Cape Provincial Research Committee.

\section{Results and actions taken}

Almost all of the 99 facilities offering TB treatment participated; only one facility in September 2008 and one in May 2009 were not audited.

Geographical access is ensured as all the facilities now offer TB diagnosis, bringing diagnosis to within $2.5 \mathrm{~km}$ of the place of residence. General access to the TB programme at facility and sub-district level was measured by the treatment commencement time (TCT) - the time from when a sputum specimen is taken to initiation of treatment (Table 1). The lengthy TCT was further investigated and delays in the laboratory process and the facility's recall of positive clients were found. An appointment card was introduced and new cadres (TB clerks and assistants) were created to improve recall of patients. These actions reduced the TCT, although it is still not under the 5-day target set.

Access for high-risk clients has improved significantly with intensified TB case finding now routine in both the HCT and ART services ( $p<0.01$ for both). This success was achieved gradually by incorporating a symptomatic screen for TB in the HCT lay counsellor training and supporting good practice with customised note-taking stationery for HCT and ART. In contrast, it has been difficult to improve the routine symptomatic screening of HIV clients attending for pre-ART care at visits; this indicator started at a higher baseline but did not improve significantly.

The sub-district HAST co-ordinators targeted access for children in contact with an infectious adult for increased mentorship support during monthly facility visits. While there was some improvement in investigating child contacts $(p=0.0013)$, this number remained disappointingly low, possibly because of the complexity of the threestep follow-up process: child contacts must be listed, and listed children must be investigated and then started on prophylaxis or treatment.

We defined quality as adherence to the local protocols and standards for TB care (Table 2). Of concern were errors noted in the transcription

Table 1. Access to TB diagnosis and treatment in Cape Town, with a focus on access for HIV-positive clients and child contacts (\% (CI))

\begin{tabular}{|c|c|c|c|c|c|c|}
\hline Indicator & March 2008 & September 2008 & May 2009 & October 2009 & August 2010 & $\begin{array}{l}p \text {-value } \\
\text { (March 2008 v. } \\
\text { August 2010) }\end{array}$ \\
\hline \multicolumn{7}{|l|}{ General } \\
\hline TB clients with TCT $<5$ days & $48(35-61)$ & $61(49-74)$ & $46(40-51)$ & $50(43-56)$ & $56(49-62)$ & 0.2843 \\
\hline \multicolumn{7}{|l|}{ Immunocompromised clients } \\
\hline $\begin{array}{l}\text { HCT clients screened } \\
\text { symptomatically for TB }\end{array}$ & $79(76-82)$ & $88(86-90)$ & $91(89-93)$ & $92(90-94)$ & $86(83-88)$ & 0.0018 \\
\hline ART clients screened sympton & & & & & & \\
\hline $\begin{array}{l}\text { for TB } \\
\text { Pre-ART clients screened }\end{array}$ & $30(24-36)$ & $59(53-64)$ & $59(54-64)$ & $67(62-72)$ & $67(63-72)$ & $<0.0001$ \\
\hline symptomatically for TB & $61(56-65)$ & $69(64-73)$ & $55(51-60)$ & $64(59-69)$ & $67(63-72)$ & 0.0465 \\
\hline \multicolumn{7}{|l|}{ Child contacts } \\
\hline $\begin{array}{l}\text { TB clients with child contacts } \\
\text { recorded }\end{array}$ & $65(52-78)$ & $51(36-67)$ & $68(61-74)$ & $69(60-77)$ & $76(70-82)$ & 0.129 \\
\hline $\begin{array}{l}\text { TB clients with child contacts } \\
\text { investigated and treated }\end{array}$ & $20(9-30)$ & $26(6-45)$ & $36(28-44)$ & $39(29-48)$ & $42(33-50)$ & 0.0013 \\
\hline
\end{tabular}




\begin{tabular}{|c|c|c|c|c|c|c|}
\hline Indicator & March 2008 & September 2008 & May 2009 & October 2009 & August 2010 & $p$-value ${ }^{*}$ \\
\hline $\begin{array}{l}\text { TB clients with data correctly transferred from } \\
\text { folder to register }\end{array}$ & $75(65-85)$ & $50(40-61)$ & $80(77-84)$ & $82(78-86)$ & $86(83-89)$ & 0.0355 \\
\hline TB clients following correct regimen & $90(83-97)$ & $80(70-90)$ & $91(88-94)$ & $94(92-96)$ & $94(92-96)$ & 0.3104 \\
\hline TB clients with sputum recall dates noted & $93(91-95)$ & $67(55-78)$ & $86(83-89)$ & $93(90-95)$ & $90(87-93)$ & 0.1400 \\
\hline $\begin{array}{l}\text { TB clients with BMI calculated (and } \\
\text { supplementation if required) }\end{array}$ & - & - & $20(16-23)$ & $47(43-51)$ & $62(58-67)$ & $<0.0001$ \\
\hline $\begin{array}{l}\text { TB clients with contraception assessed (men and } \\
\text { women) }\end{array}$ & - & $27(19-35)$ & $41(36-45)$ & $51(46-56)$ & $58(53-63)$ & $<0.0001$ \\
\hline $\begin{array}{l}\text { TB clients who had HIV test done (HCT offered } \\
\text { 2007) }\end{array}$ & $93(91-95)$ & $89(83-96)$ & $92(90-94)$ & $92(90-95)$ & $96(94-98)$ & 0.0882 \\
\hline $\begin{array}{l}\text { Co-infected TB/HIV clients for whom HIV } \\
\text { stationery was used }\end{array}$ & $51(36-66)$ & $29(17-41)$ & $54(47-61)$ & $67(61-73)$ & $79(73-85)$ & 0.0006 \\
\hline $\begin{array}{l}\text { Co-infected TB/HIV clients who had a CD4 count } \\
\text { and staging done }{ }^{\dagger}\end{array}$ & $58(43-72)$ & $30(18-41)$ & - & - & - & Not done \\
\hline $\begin{array}{l}\text { Co-infected TB/HIV clients who had a CD4 count } \\
\text { done }^{\dagger}\end{array}$ & - & - & $92(89-96)$ & $91(88-95)$ & $92(88-96)$ & Not done \\
\hline $\begin{array}{l}\text { Co-infected TB/HIV clients who were staged } \\
\text { according to WHO criteria* }\end{array}$ & - & - & $76(56-95)$ & $58(36-79)$ & $71(53-89)$ & Not done ${ }^{*}$ \\
\hline $\begin{array}{l}\text { Co-infected TB/HIV clients who received } \\
\text { co-trimoxazole prophylaxis }\end{array}$ & $88(83-92)$ & $81(71-92)$ & $80(75-86)$ & $81(76-86)$ & $89(84-94)$ & 0.7392 \\
\hline
\end{tabular}

of routine programme data from the clients' folders (the primary data source) to the paper-based register from which the data are then re-entered into the electronic TB register. However, folder reviews mostly showed that, where quality of care for TB was high at baseline, this was sustained; and where lower at baseline, it improved significantly. Case holding was improved by training and mentoring staff in the TB treatment rooms in each facility to record the anticipated dates for the repeat sputum examination and for change to the continuation phase in the patient's folder during the initial assessment. A locally developed treatment wheel ${ }^{6}$ allowed quick calculation of these dates. Nutritional therapy is a crucial adjunct in treating TB in malnourished patients. In May 2009, the body mass index (BMI) of only $20 \%$ of TB patients was measured; this improved to $62 \%$ after protocol training and providing weighing scales and BMI calculation wheels for each $\mathrm{TB}$ room. Assessment of contraception requirements for men and women TB patients improved from 27\% in September 2008 to 58\% in August 2010 with mentoring on integrated HAST guidelines.

High levels of HCT (93\%) were achieved within the TB programme since March 2008. The use of customised HIV stationery with quality prompts for co-infected clients improved significantly $(p=0.0006)$. Assessing TB patients for ART improved, though an indicator change makes significance testing impossible (58\% of co-infected clients had a CD4 count and staging done in March 2008; 92\% had a CD4 count done and 71\% were staged clinically in August 2010).

\section{Discussion}

Good-quality, timeous routine data are essential to manage the TB programme effectively. ${ }^{8}$ Routine information is limited in scope and open to errors in transcription from folder to paper registers to electronic database. ${ }^{9}$ The present study confirmed this. The audit validated and supplemented the routine data. Where there had been large gains in improving cure rates over the last 5 years, sub-district and facility managers might have been satisfied that the programme had strengthened overall and that the $85 \%$ success rate target was within reach. Nevertheless, the use of a clinical audit provided more details of access to and quality of the TB programme, and identified gaps in effectiveness to be addressed.

Early case detection in a TB control programme to reduce the infectious load in the community is critical and relies on prompt access to the health service. ${ }^{8}$ The WHO indicator for access is the case detection rate, where the numerator is the number of $\mathrm{TB}$ patients started on treatment and the denominator is the total number of people estimated to have TB. The latter is modelled from national prevalence studies and vital statistics. While the indicator can be calculated at national level (and the National Strategic Plan ${ }^{3}$ has set a target of 70\%), it is not feasible to assess the indicator at district or facility level. The clinical audit partly addressed this gap and provided measures that could be addressed by action at facility and sub-district level. The TCT is a useful local measure, and sub-district and facility managers could identify local health system interventions to reduce delays. Health system constraints are significant causes of delays in TB diagnosis and treatment. ${ }^{10} \mathrm{~A}$ South African study ${ }^{11}$ found provider-related delay to be double patient-related delay. The audit, in its selection of indicators, also promoted early case detection in HIV-positive patients, and highlighted deficiencies in access for child contacts. To improve access in high-prevalence communities such as South Africa further, community-based case finding should be considered, ${ }^{12}$ with point-ofcare diagnostics for sputum-positive and -negative clients. ${ }^{13}$ 


\section{RESEARCH}

The improved cure rate outcome is the most important measure of improved quality of TB care in the Cape Town TB programme. The audit reinforced the treatment guidelines and standard operating procedures, and provided HAST co-ordinators with a mentoring opportunity. Quality gains were evident in the HIV care for co-infected patients, BMI calculation and contraception assessment rates. These 'quick wins' demonstrated the benefits of measuring key activities to determine whether protocols are being followed, and of engaging staff in problem solving to identify constraints and plan improvements in service delivery. The participatory audit process involving facility and sub-district staff in self- and peer-review, and generating facility and sub-district results, drove a quality improvement process that was local and relevant. Other studies also found this approach to be effective. ${ }^{14,15}$ Audit workshops and quarterly monitoring and evaluation sessions created opportunities for the transfer and sharing of best practice among managers and staff.

Developing and using the HAST audit tools was only one of the Cape Town strategies to improve TB cure rates. Synergy between the strategies enhanced their effectiveness.

The facility non-response rate was small (1.1\%) and we do not anticipate significant bias. The interventions used in Cape Town are not necessarily appropriate or sustainable in other districts; rather it is the participatory process that has potential for replication.

Acknowledgements. This paper was written on behalf of the provincial HIV/TB task team who developed the tools, oversaw the audits and facilitated feedback to sub-districts. Juanita Arendse, Virginia Azevedo, Judy Caldwell, Karen Jennings, Pren Naidoo, Vera Scott, Brenda Smuts, Alvera Swartz and Virginia Zweigenthal contributed significantly to the work on TB. We thank Hilton Snyder and Professor Richard Madsen for support in data management and analysis, and the sub-district HAST co-ordinators, sub-district/sub-structure managers, facility managers and staff.

\section{References}

1. World Health Organization. Global Tuberculosis Control 2010. Geneva: WHO, 2010.

2. Barr D, Padarath A, Sait L. The Stop TB Partnership in South Africa - a Review. Durban: Health Systems Trust, 2005

National Department of Health. Tuberculosis Strategic Plan for South Africa, 2007 - 2011. Pretoria National Department of Health, 2008.

4. Schneider H, Barron P, Fonn S. The promise and practice of transformation in South Africa's health system. In: Buhlungu S, Daniel J, Southall R, Lutchman J, eds. State of the Nation, South Africa 2007. Cape Town: Human Sciences Research Council, 2007.

5. Edginton M, Naidoo S. Tuberculosis: a deepening crisis in South Africa. Southern African Journal of Epidemiology and Infection 2007;22(2):37-38

6. Scott VE, Chopra M, Azevedo V, Caldwell J, Naidoo P, Smuts B. Scaling up integration: developmen and results of a participatory assessment of HIV/TB services, South Africa. Health Research Policy and Systems 2010;8,23.

7. Caldwell I. The TB treatment wheel: An aid to move from 'treatment completion' to 'cure. Conference abstract, 38th Union World Conference of Lung Health, 9-12 November 2007, Cape Town.

8. World Health Organization. The Stop TB Strategy: Building on and Enhancing DOTS to Meet the TBrelated Millennium Development Goals. Geneva: WHO, 2006

9. Norval P-Y, Heldal E, L'Herminez R, Laserson K, Godfrey A. Revising the tuberculosis recording and reporting information system. Int J Tuberc Lung Dis 2008;12(3):S17-S19.

10. Storla DG, Yimer S, Bjune GA. A systematic review of delay in the diagnosis and treatment of tuberculosis. BMC Public Health 2008;8:15. [http://dx.doi.org/10.1186/1471-2458-8-15]

11. Meintjes G, Schoeman H, Morroni C, Wilson D, Maartens G. Patient and provider delay in tuberculosis suspects from communities with a high HIV prevalence in South Africa: a cross-sectional study $\mathrm{BMC}$ Infect Dis 2008;8:72-79. [http://dx.doi:10.1186/1471-2334-8-72]

12. Wood R, Lawn SD, Johnstone-Robertson S, Bekker, L-G. Tuberculosis control has failed in South Africa - time to reappraise strategy. HIV Medicine 2011;101(2):110-114.

13. Wallis RS, Pai M, Menzies D, et al. Biomarkers and diagnostics for tuberculosis: progress, needs, Wallis RS, Pai M, Menzies D, et al. Biomarkers and diagnostics for tuberculosis: progress, needs,
and translation into practice. Lancet 2010;375(9729):1920-1937. [http://dx.doi.org/10.1016/S01406736(10)60359-5

14. Kanara N, Cain KP, Laserson KF, et al. Using program evaluation to improve the performance of a TBHIV project in Banteay Meanchey, Cambodia. Int J Tuberc Lung Dis 2008;12(3):S44-S50.

15. Doherty T, Chopra M, Nsibande D, Mngoma D. Improving the coverage of the PMTCT programme Doherty T, Chopra M, Nsibande D, Mngoma D. Improving the coverage of the PMTCT programme
through a participatory quality improvement intervention in South Africa. BMC Public Health 2009; 9:406. [http://dx.doi.org/10.1186/1471-2458-9-406]

Accepted 10 February 2012. 